\title{
Facilitation of endotracheal intubation using a bougie in a patient with unilateral vocal cord due to a traumatic thyroid cartilage fracture
}

\author{
Eun-A Jang ${ }^{1}$, Seung Myung Park', Seongheon Lee ${ }^{1,2}$, Joungmin Kim ${ }^{1,2}$ \\ Department of Anesthesiology and Pain Medicine, ${ }^{1}$ Chonnam National University Hospital, ${ }^{2}$ Chonnam National University Medical \\ School, Gwangju, Korea
}

Received July 14, 2021

Revised July 29, 2021

Accepted August 4, 2021

Corresponding author Joungmin Kim

Department of Anesthesiology

and Pain Medicine, Chonnam

National University Medical School,

Chonnam National University

Hospital, 42 Jebongro, Dong-gu,

Gwangju 61469, Korea

Tel: +82-62-220-6895

Fax: +82-62-232-6294

E-mail: tca77@hanmail.net

ORCID:

https://orcid.org/0000-0003-1135-1968
Endotracheal intubation of patients with abnormal upper airway structures is rare. In these patients, endotracheal intubation may be difficult and sometimes emergent surgical airway security may be required, so a thorough airway evaluation, intubation plan, and implementation should be performed. We report a case of successful endotracheal intubation using bougie in the patient with unilateral vocal cord and narrow glottis who underwent thyroid cartilage fracture 10 years ago.

Keywords: Airway management; Intubation; Larynx; Thyroid cartilage

\section{INTRODUCTION}

Laryngeal injuries due to trauma have still a low prevalence, although it is increasing recently [1,2]. Laryngeal injuries associated with fractures are life-threatening events that sometimes require emergent intervention. In the treatment of laryngeal fractures, securing the airway is prioritized in the early stage, and in the later stage, the voice related to quality of life is considered [3]. Destruction and regeneration of laryngeal structures occurs during this procedure, and changes in these structures are challenging for anesthesiol- ogists because of the potential for difficult airway management. Laryngeal injuries have been mainly reported in the forms of case reports and medical record reviews on its initial management, and there have been only a few reports on their long-term prognosis. In particular, there have not been reports on the airway management for patients undergoing general anesthesia who have suffered laryngeal injuries in the past.

Herein, we describe a case of successful endotracheal intubation using a bougie in the patient with unilateral vocal cord due to a traumatic thyroid cartilage fracture. 


\section{CASE REPORT}

A 70-year-old female patient $(154 \mathrm{~cm}, 45 \mathrm{~kg})$ was scheduled to undergo general anesthesia for open reduction and internal fixation of a left humerus head fracture that occurred after slip-down. She had a history of thyroid cartilage repair due to a car accident 10 years ago and since then, had complained of mild dyspnea and hoarseness. Preoperative neck computed tomography (Fig. 1) scan showed partial absorption of thyroid cartilage and cricoid cartilage and mild contour deformation of larynx with luminal patency. In addition, preoperative laryngeal fiberscopy (Fig. 2) showed a defect in the left larynx and vocal cord, which was replaced by soft tissue, and only her right vocal cord remained with stricture of glottic inlet which suggesting that endotracheal intubation may be difficult. Other upper airway assessments (neck flexion and extension, mouth opening and dental conditions) did not show any specific findings. We decided to use videolaryngoscopy for difficult endotracheal intubation, and bougie for possibility of multiple attempts of endotracheal intubation to determinate appropriately sized endotracheal tube.

On the day of surgery, standard monitoring, including electrocardiography, noninvasive blood pressure monitoring, capnography, end-tidal vapor analysis, and pulse oximetry, and bispectral index were used in the operating room. After preoxygenation with $100 \%$ oxygen, anesthesia was in-

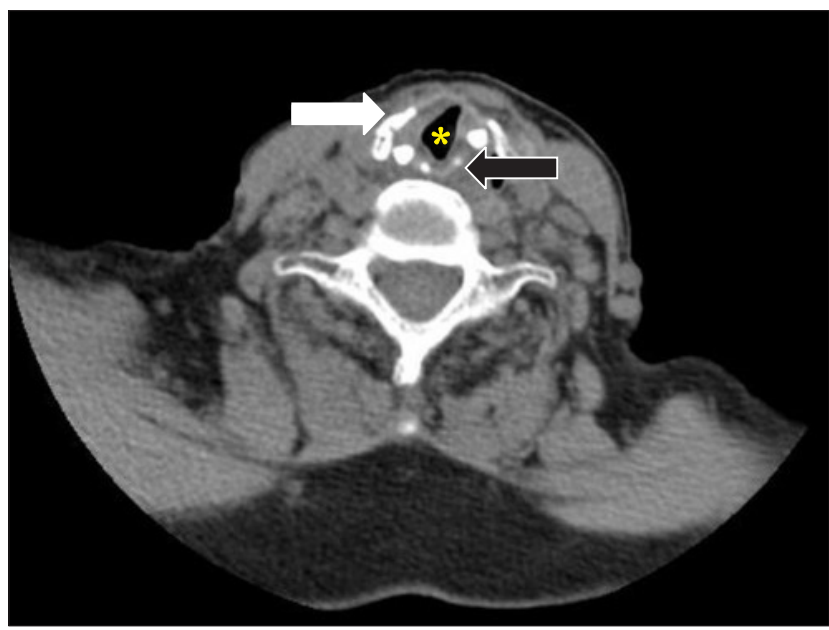

Fig. 1. Neck CT image shows partial absorption of thyroid cartilage (white arrow) and cricoid cartilage (black arrow), contour deformity of larynx with luminal patency $(*)$. duced with intravenous administration of propofol $(1.5 \mathrm{mg} /$ $\mathrm{kg})$, remifentanil $(1 \mathrm{mcg} / \mathrm{kg})$, and after confirming that the mask was well ventilated, rocuronium $(0.8 \mathrm{mg} / \mathrm{kg})$ was administered. The patient was ventilated for 3 min with $100 \%$ oxygen and sevoflurane. We visualized the glottic structures with KoMAC videolaryngoscope (KoMAC Co., Ltd, Seoul, Korea), and inserted the bougie past the vocal cords into the trachea (Fig. 3A and 3B). Then we tried endotracheal intubation with endotracheal tube size of 6.0 over the bougie, but there was some resistance, so we downsized endotracheal tube to 5.5 while maintaining the bougie (Fig. 3C). In endotracheal tube size of 5.5, endotracheal intubation was performed without resistance, and the bougie was withdrawn from the endotracheal tube. After successful tracheal intubation, anesthesia and surgery progressed well. The operation times were approximately 2 hours and 30 minutes. After surgery, the patient was able to obey simple commands and the train-of-for (TOF) ratio of 0.9 or higher was checked, and then endotracheal extubation was performed uneventfully. On arrival at the postanesthesia care unit, the oxygen saturation was $95 \%$ given the $\mathrm{O}_{2}$ mask support $(5 \mathrm{~L} /$ $\mathrm{min}$ ), and after that, the respiratory pattern was kept stable and the patient was transferred to the ward. There were no abnormalities on the chest radiograph after surgery, and there were no specific problems in the ward, so the patient was discharged 5 days after surgery.

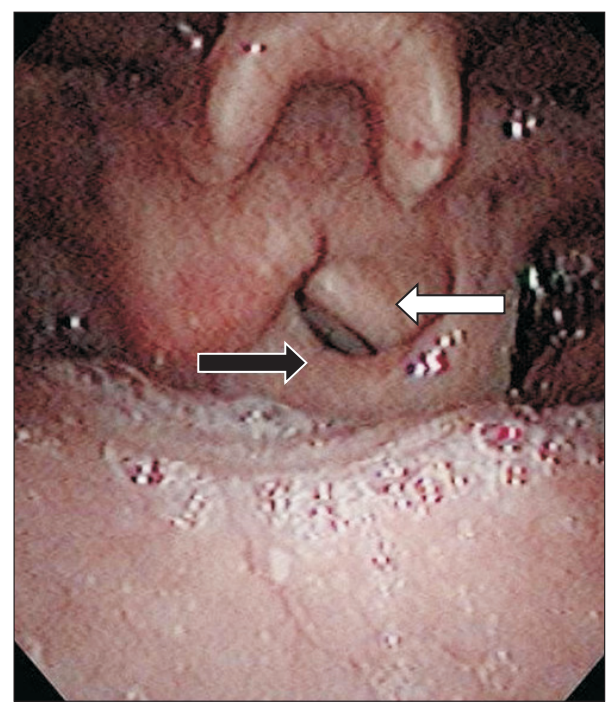

Fig. 2. Laryngeal fiberscopy shows that defect of left larynx and vocal cord (black arrow) which was replaced by soft tissue, only remained right vocal cord (white arrow), and stricture of glottic inlet. 


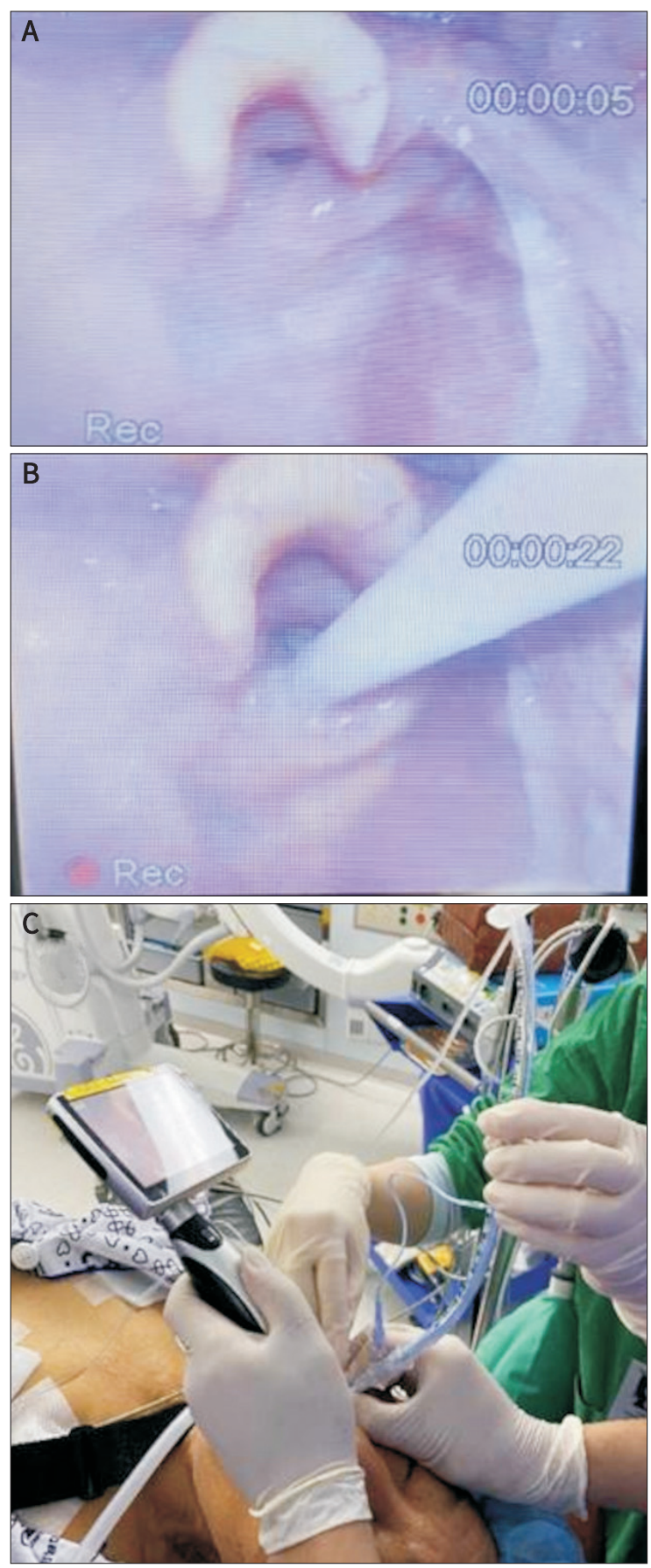

Fig. 3. Images from (A) glottic structures visualized with videolaryngoscopy, (B) insertion of the bougie through vocal cords into trachea, (C) endotracheal intubation with endotracheal tube over the bougie.

\section{DISCUSSION}

Laryngeal injuries are major risk factors for difficult endotracheal intubation. In the case of laryngeal injuries, it is difficult to obtain profer laryngeal view due to epiglottis and displaced laryngeal inlet caused by hematoma, laryngeal edema, or fractures [4,5]. Therefore, it may be emergently needed cricothyroidotomy or tracheotomy. Our patient was also taken to the emergency room right after the accident 10 years ago and tried endotracheal intubation to secure an airway, however, due to failure to secure an airway, a tracheotomy was performed.

In addition to laryngeal injuries, loss of normal upper airway anatomy may also cause endotracheal intubation failure. According to a retrospective review, more than $50 \%$ of patients with difficult airway structures require surgical airway access due to tracheal intubation failure [6]. Therefore, caution should be undertaken when managing airway in patients with history of laryngeal injuries because there may be changes in the airway structure.

In this case, the patient had sustained laryngeal injury from a car accident 10 years ago and had undergone tracheostomy and thyroid cartilage repair. Regional anesthesia could be considered, but the possibility of ipsilateral phrenic nerve block could not be excluded if interscalene brachial plexus block was performed for proximal shoulder surgery [7]. In normal patients, this would not be a problem, but in this patient, because of the abnormality of the airway structure, it was judged that there is a risk of respiratory support not well if hypoxia or dyspnea would occur. Therefore, we decided to put the patient under general anesthesia, and because endotracheal intubation was expected to be difficult, various examinations were performed to accurately evaluate the airway. The patient had only a unilateral vocal cord, and it was confirmed that laryngeal structures were deformed, such as a narrowed glottic inlet. Thorough planning was required to avoid damage to the deformed laryngeal structures during endotracheal intubation.

Laryngeal complications due to endotracheal intubation include hyperemia, edema, local scarring, rare bleeding, perforation, arytenoid dislocation, and vocal cord paralysis etc [8-10]. These laryngeal complications occur more frequently during difficult endotracheal intubation because of unusual anatomy, following the use of an introducer, unskilled intubation, or after repeated intubation [8]. There are reports that the success of endotracheal intubation on the first attempt reduces these complications and surgical intervention such as cricothyroidotomy or tracheotomy [11,12]. Further damage could have been fatal to our patient, therefore we tried to minimize the number of endotracheal intu- 
bation attempts and to perform it as smoothly as possible. In order to pass through the narrowed airway and deformed laryngeal structure, judicious selection of appropriate sized endotracheal tubes and adjunctive devices was required.

There are no guidelines for endotracheal tube size selection, but in adults, it is usually determined according to the patient's height or sex [13]. The endotracheal tube with larger inner diameter may be maximized ventilation and reduced work of breathing, but can sometimes be difficult to intubate or damage the upper airway. The endotracheal tube with smaller inner diameter is less likely to damage the upper airway, but ventilation may be insufficient or cuff pressure may need to be increased to maintain an adequate seal [14]. We decided to use the 6.0 tube first, considering that the patient is a woman and her height, and reduced the size if resistance was felt. In the process of endotracheal tube downsizing, we tried to perform endotracheal intubation as smoothly as possible using a bougie because of the possibility that multiple intubation attempts could be made unnecessarily.

Usually, stylet and bougie are as endotracheal intubation introducers, and the two devices have slightly different intubation processes. The stylet passes into the trachea with endotracheal tube, but the bougie first passes through the trachea and then endotracheal tube advances over the bougie. Several studies have reported that the use of bougie increased the success rate of difficult endotracheal intubation [15,16, and the success rate of intubation on the first attempt was higher in the group using the bougie than in the group using the stylet [17-19]. Latto et al. [15] reported that it is important to obtain at least the best view of the larynx when using the bougie, but according to Cook [20], the use of a bougie showed a $94.3 \%$ success rate even when the larynx was not visible. These results can be explained by the fact that the flexibility of the bougie made it easier to pass through the anterior wall of the larynx into the trachea [18], and its smaller diameter less obstructed the operator's view when passing through the narrow glottis. The bougie can enter under the epiglottis to the trachea blindly when the glottic view is incomplete (Cormack-Lehane grade 2-4), and provides tactile feedback when the bougie tip reaches the bronchial tree [19]. It is thought that each clinician's opinion will differ as to which auxiliary device to select for the first intubation attempt. In our patient, the epiglottis tip was visualized by preoperative laryngeal fibroscopy, and also there was possibility of tube size change, so the seleciton of the bougie would be reasonable. Therefore, we were able to successfully perform endotracheal intubation in the patient with unilateral vocal cord and narrow glottis using the bougie.

In conclusion, it is safe to select an appropriate endotracheal tube size and perform bougie-guided endotracheal intubation in patients with upper airway deformities such as stenosis, and unilateral vocal cord, and this can reduce the incidence of complications.

\section{CONFLICT OF INTEREST}

No potential conflict of interest relevant to this article was reported.

\section{REFERENCES}

1. Jalisi S, Zoccoli M. Management of laryngeal fractures--a 10year experience. J Voice 2011;25:473-9.

2. VandeGriend ZP, Hashemi A, Shkoukani M. Changing trends in adult facial trauma epidemiology. J Craniofac Surg 2015; 26:108-12.

3. Kim JP, Cho SJ, Son HY, Park JJ, Woo SH. Analysis of clinical feature and management of laryngeal fracture: recent 22 case review. Yonsei Med J 2012;53:992-8.

4. Flood LM, Astley B. Anaesthetic management of acute laryngeal trauma. Br J Anaesth 1982;54:1339-43.

5. Jain U, McCunn M, Smith CE, Pittet JF. Management of the traumatized airway. Anesthesiology 2016;124:199-206.

6. Stephens CT, Kahntroff S, Dutton RP. The success of emergency endotracheal intubation in trauma patients: a 10-year experience at a major adult trauma referral center. Anesth Analg 2009;109:866-72.

7. El-Boghdadly K, Chin KJ, Chan VWS. Phrenic nerve palsy and regional anesthesia for shoulder surgery: anatomical, physiologic, and clinical considerations. Anesthesiology 2017;127:173-91.

8. Benjamin B. Laryngeal trauma from intubation: endoscopic evaluation and classification. In: Cummings CW, Fredrickson JM, Harker LA, Krause CJ, Schuller DE, editors. Otolaryngology head and neck surgery. 2nd ed. St. Louis: Mosby; 1993. p. 1875-96.

9. Xu W, Han D, Hu R, Bai Y, Zhang L. Characteristics of vocal fold immobility following endotracheal intubation. Ann Otol Rhinol Laryngol 2012;121:689-94.

10. Chun EH, Baik HJ, Chung RK, Lee HJ, Shin K, Woo JH. Ar- 
ytenoid cartilage dislocation mimicking bilateral vocal cord paralysis: A case report. Medicine (Baltimore) 2017;96:e8514.

11. Mort TC. Emergency tracheal intubation: complications associated with repeated laryngoscopic attempts. Anesth Analg 2004;99:607-13.

12. Sakles JC, Chiu S, Mosier J, Walker C, Stolz U. The importance of first pass success when performing orotracheal intubation in the emergency department. Acad Emerg Med 2013;20:71-8.

13. Cao AC, Rereddy S, Mirza N. Current practices in endotracheal tube size selection for adults. Laryngoscope 2021;131: 1967-1.

14. Schiff BA. The relationship between body mass, tracheal diameter, endotracheal tube size, and tracheal stenosis. Int Anesthesiol Clin 2017;55:42-51.

15. Latto IP, Stacey M, Mecklenburgh J, Vaughan RS. Survey of the use of the gum elastic bougie in clinical practice. Anaes- thesia 2002;57:379-84.

16. Jabre P, Combes X, Leroux B, Aaron E, Auger H, Margenet A, et al. Use of gum elastic bougie for prehospital difficult intubation. Am J Emerg Med 2005;23:552-5.

17. Kidd JF, Dyson A, Latto IP. Successful difficult intubation. Use of the gum elastic bougie. Anaesthesia 1988;43:437-8.

18. Gataure PS, Vaughan RS, Latto IP. Simulated difficult intubation. Comparison of the gum elastic bougie and the stylet. Anaesthesia 1996;51:935-8.

19. Driver BE, Prekker ME, Klein LR, Reardon RF, Miner JR, Fagerstrom ET, et al. Effect of use of a bougie vs endotracheal tube and stylet on first-attempt intubation success among patients with difficult airways undergoing emergency intubation: a randomized clinical trial. JAMA 2018;319:2179-89.

20. Cook TM. A new practical classification of laryngeal view. Anaesthesia 2000;55:274-9. 